\title{
Underthreshold resonances in three-particle molecular systems
}

\author{
F.M. Pen'kov \\ Joint Institute for Nuclear Research, \\ 141980, Dubna, Russia \\ penkov@thsun1.jinr.ru
}

\begin{abstract}
To determine the lifetimes of Efimov states of negative two-atomic ions, the problem of resonance scattering of a light particle on a pair of identical particles has been considered. An analytic expression has been obtained for resonance widths in the limit of forces of zero radius and low binding energies in pairs. Calculations are compared with the numerical solution of the Faddeev integral equations in a wide region of masses of the light particle. It is shown that the widths of underthreshold resonances in the scattering amplitude obtained from the integral equations with the Yamaguchi potential are well described by the analytic expression, which allows this expression to be used in the mass region inaccessible for numerical calculations. It has been concluded that the lifetime of highly excited negative molecular ions with a binding energy close to the threshold of disintegration is practically unlimited.
\end{abstract}

\section{Introduction}

Recent papers [1, 2, 3] have dealt with the exotic states of systems of neutral atoms and an electron. These states are characterized by large (tens of angstroms) sizes and demonstrate the effects of three-body dynamics at low binding energies in pairs. In particular, a direct analysis [1] of the Faddeev equations allowed us to determine the effective potential of interaction of two neutral atoms in the presence of an electron. This potential is local in the rangef

$$
\max \left(r_{0}\right) \ll r \ll \min \left(\kappa^{-1}\right)
$$

( $r_{0}$ is the range of two-body forces and $\kappa$ is the wave number of a bound or virtual state of a pair) and contains both the long-range components of the type $1 / r^{2}$ typical for the Efimov effect [4] and quasi-Coulomb terms of the type $1 / r$ that essentially contribute to the spectrum of negative two-atomic molecular ions with a small binding energy (real

\footnotetext{
${ }^{1}$ Here the Planck constant $\hbar=1$
} 
or virtual) of an electron and an atom. Specifically, experimental data on the scattering of an electron by a helium atom allow us to conclude on a possible existence of a bound state of the system $\mathrm{He}_{2}^{-}$even when there is no atom-atomic interaction [2]. Moreover, the analysis of a system of three neutral atoms of alkali metals and an electron [3] shows that the effective interaction generated by the electron produce more than thousand bound states. It has been assumed that similar systems can initiate clusters in highly rarefied gases. However, that analysis is not complete for the atom- atomic interaction with the binding energy higher than that of a three-particle system. In this case, the system can decay into a molecule and a free electron, and bound states of the threeparticle system can transform into resonances with the lifetime determined by widths of these resonances.

The present paper is devoted to the study of these widths in the framework of the three-particle problem of scattering of a light particle on a bound pair of heavy particles. Like in refs. [1, 2], we will consider the systems in which the lengths of twobody scattering are much larger than the effective ranges of two-body forces satisfying the conditions of the Efimov effect:

$$
\kappa r_{0} \ll 1
$$

The binding energy of atoms is taken finite; whereas that in electron-atom pairs, zero for simplifying, analytic computations. The threshold of rearrangement of the system coincides with the threshold of disintegration and the above-mentioned spectrum of three-particle systems [1, 2] is manifested as underthreshold Efimov resonances. Resonances of that sort, but under the excitation threshold, in a three-boson system were considered in ref.[5] and equidistance was shown both for positions of resonances and their widths on the logarithmic scale.

In this paper, we consider the case when the mass of one particle is considerably smaller than the masses of two other particles. Here we are faced with almost classical motion of heavy particles [1], which does not allow accurate numerical solution of the Faddeev equations for the ratios of masses of an atom and an electron. Therefore, like in ref.[1], numerical calculations are carried out for the ratios of masses not exceeding $1 / 100$ in order to verify the analytic relation between the width and position of a resonance that can be derived at extremely small effective ranges of two-body forces. It is demonstrated below that the analytic expression satisfactorily describes a highly complicated mass dependence of the resonance widths, and thus it can be used to estimate the resonance widths for realistic ratios of electron-atomic masses.

\section{Integral equations}

We consider a system of three spinless particles with masses $m_{i}$, where the index $i=1,2,3$ denotes a particle, and with two-body potentials $v_{i}$, whose index corresponds to a particle absent in a two-particle subsystem. Identical particles 2 and 3 with masses $m_{2}=m_{3}=m$ interact via the potential $v_{1}$ and form a bound state with the energy $\varepsilon_{1}=-\kappa_{1}^{2} / 2 m_{23}$, where $m_{i j}$ is the reduced mass of particles $i$ and $j$. Particle 1 with mass $m_{1}$ interact with particles 2 and 3 via the potentials $v_{3}$ and $v_{2}\left(v_{2}=v_{3}=v\right)$, 
respectively, which generate two-particle states (real or virtual) with an extremely low binding energy $\left(\kappa_{2}=\kappa_{3}=\kappa \rightarrow 0\right)$. The potentials $v_{i}$ (between particles $\mathrm{j}$ and $\mathrm{k}$ ) were taken to be the separable Yamaguchi potentials acting only in the s-wave:

$$
v_{i}\left(p, p^{\prime}\right)=-\frac{4 \pi}{m_{j k}} \frac{\beta_{i}\left(\beta_{i}+\kappa_{i}\right)^{2}}{\left(\beta_{i}^{2}+p^{2}\right)\left(\beta_{i}^{2}+{p^{\prime}}^{2}\right)} .
$$

The sign of the wave number $\kappa$ defines either a bound $(\kappa>0)$ or a virtual state of a pair. The parameter $\beta_{i}$ determines the range of forces in a pair. $i$. When $\beta_{i} \gg \kappa_{i}$, the expansion of the effective range in a pair $i$ over the momentum of relative motion $p$ $\left(p \operatorname{cotan}\left(\delta_{i}\right)=\kappa_{i}+r_{e f f} p^{2} / 2+\ldots\right)$ gives $r_{e f f}=3 / \beta_{i}$, which allows us to employ condition (2) in the form $\kappa_{i} \ll \beta_{i}$. In what follows for brevity, we use the separable potential given by projectors: $v_{i}=\left|\nu_{i}><\nu_{i}\right|$.

Consider the scattering of particle 1 on a bound state of identical particles 2 and 3 with the energy of relative motion $E_{k}=k_{0}^{2} / 2 m_{1,23}$, lower than the energy of rearrangement of the system $\varepsilon_{2}-\varepsilon_{1}$. Here $m_{i, j k}$ is the reduced mass of particle $\mathrm{i}$ and a pair $(\mathrm{j}, \mathrm{k})$. The momentum $\mathbf{k}_{\mathbf{i}}$ is always the momentum of relative motion of particle $\mathrm{i}$ and pair $(\mathrm{j}, \mathrm{k})$, whereas the momentum $\mathbf{p}_{\mathbf{i}}$ refers to motion inside the pair $(\mathrm{j}, \mathrm{k})$. (If no confusion, the indices are omitted.)

To write the system of Faddeev integral equations (see, e.g., [6]), we need an expression for the product of a two-body t-matrix in the 3-particle space and the free Green function $G^{0}(Z)=\left(Z-h_{i}^{0}-h_{(i)}^{0}\right)^{-1}$. The Hamiltonian of free motion of three particles is given by two terms, the Hamiltonian of free motion $h_{i}^{0}$ of particle i and pair $(\mathrm{j}, \mathrm{k})$ and the Hamiltonian of relative motion in pair $h_{(i)}^{0}$. The quantity $Z=E_{k}+\varepsilon_{1}+i 0$ is the total energy of a 3-particle system. For separable potentials, this product can be represented in the form [7]

$$
t G^{0}(Z)=v_{i}\left|\varphi_{i}>g_{i}^{0}\left(Z-\varepsilon_{i}\right)<\tilde{\varphi}_{i}\right|
$$

that includes the wave function of a two-particle bound state $\left|\varphi_{i}\right\rangle$ and the function $<\tilde{\varphi}_{i}(Z) \mid$ defined in the 3-particle space. Hereafter, we make use of expressions for 2particle Green functions $g_{i}^{0}(x)$ and $g_{(i)}^{0}(x)$ corresponding to two-particle Hamiltonians $h_{i}^{0}$ and $h_{(i)}^{0}$, respectively. In this notation, the projection of the function $<\tilde{\varphi}_{i} \mid$ on $<\mathbf{k}_{\mathbf{i}} \mid$ is of the simple form:

$$
\begin{gathered}
<\tilde{\varphi}_{i}\left|=R_{i}<\varphi_{i}\right| v_{i} G^{0}\left(Z-k_{i}^{2} / 2 m_{i, j k}\right) \\
R_{i}=-\frac{1}{2 m_{j k}} \frac{\left(\beta_{i}+a_{i}\right)^{2}\left(a_{i}+\kappa_{i}\right)}{\left(2 \beta_{i}+\kappa_{i}+a_{i}\right)<\varphi_{i}\left|v_{i}\right| \varphi_{i}>} ; \quad a_{i}=\sqrt{-2 m_{j k}\left(Z-k_{i}^{2} / 2 m_{i, j k}\right)} .
\end{gathered}
$$

Note that the 2-particle $t$-matrix corresponding to a separable potential of the Yamaguchi type has two poles. The near one is at the point of a 2-particle bound state, $p_{i}= \pm i \kappa_{i}$ (on the physical sheet for a realistic bound state and on the unphysical sheet for a virtual state). The distant pole on the unphysical sheet $p_{i}=-i\left(2 \beta_{i}+\kappa_{i}\right)$ determines the range of two-body forces. The limit of zeroth range is achieved with $\beta$ tending to infinity. In this limit, there remains one pole, and therefore it does not matter, which two-body potential, either local or nonlocal, has generated it. Here we 
use the separable potential only for reasons of simplification of writing the Faddeev integral equations. In this case, just $t$-matrices remain finite in the limit $\beta \rightarrow \infty$.

Once the two-particle $t$-matrices are determined, we write the Faddeev equations for the scattering of particle 1 on a bound pair of identical particles 2 and 3 in the form

$$
\begin{gathered}
T^{e l}=V_{12} g_{2}^{0}\left(Z-\varepsilon_{2}\right) T^{r} \\
T^{r}=2 V_{21}+2 V_{21} g_{1}^{0}\left(Z-\varepsilon_{1}\right) T^{e l}+V_{23} g_{3}^{0}\left(Z-\varepsilon_{3}\right) T^{r}
\end{gathered}
$$

where $V_{i j}=R_{i}^{1 / 2}<\varphi_{i}\left|v_{i} G_{0}(Z) v_{j}\right| \varphi_{j}>R_{j}^{1 / 2}$, and the transition matrices $T^{e l}$ and $T^{r}$ are connected with the physical amplitudes of elastic and inelastic scattering by the simple formulae:

$$
f^{e l}=-\frac{m_{1,23}}{2 \pi} T^{e l}\left(\mathbf{k}_{1}^{\text {out }}, \mathbf{k}_{1}^{\text {in }}\right), \quad f^{r}=-\frac{\sqrt{m_{1,23} m_{2,13}}}{2 \pi} T^{r}\left(\mathbf{k}_{2}^{\text {out }}, \mathbf{k}_{1}^{\text {in }}\right),
$$

where in- and out-momenta $k^{\text {in }}$ and $k^{\text {out }}$ are on energy surfaces.

The system of complex integral equations (5) can be transformed into the system of equations real up to the threshold of rearrangement (see, e.g., [8]). The simple change upon partial decomposition

$$
\begin{gathered}
f^{e l}\left(k, k_{0}\right)=-\frac{m_{1,23}}{2 \pi} K^{e l}\left(k, k_{0}\right)\left(1+i k_{0} f^{e l}\left(k_{0}, k_{0}\right)\right), \\
f^{r}\left(k, k_{0}\right)=-\frac{\sqrt{m_{1,23} m_{2,13}}}{2 \pi} K^{r}\left(k, k_{0}\right)\left(1+i k_{0} f^{e l}\left(k_{0}, k_{0}\right)\right)
\end{gathered}
$$

results in the system of equations for real functions $K^{e l}$ and $K^{r}$ solved numerically. To distinguish the real equations with principal-value integrals, we further denote the total energy as $z=\operatorname{Re} Z$. In this notation, real integral equations for $K^{e l}$ and $K^{r}$ coincide with eqs. (5) when $T^{e l}$ is replaced by $K^{e l} ; T^{r}$, by $K^{r}$; and $Z$, by $z$, respectively:

$$
\begin{gathered}
K^{e l}=V_{12} g_{2}^{0}\left(z-\varepsilon_{2}\right) K^{r} \\
K^{r}=2 V_{21}+2 V_{21} g_{1}^{0}\left(z-\varepsilon_{1}\right) K^{e l}+V_{23} g_{3}^{0}\left(z-\varepsilon_{3}\right) K^{r} .
\end{gathered}
$$

Just these equations for $K^{e l}$ and $K^{r}$ were solved numerically. Below, we show the scheme of origin of underthreshold resonances on the basis of equations (7).

\section{Underthreshold resonances}

To see the origin of underthreshold resonances, we transform eqs. (7) as follows:

$$
\begin{gathered}
K^{e l}=V_{12} g_{2}^{0}\left(z-\varepsilon_{2}\right) K^{r}, \\
K^{r}=2 V_{21}+V g_{3}^{0}\left(z-\varepsilon_{3}\right) K^{r},
\end{gathered}
$$

where the effective potential of an (inelastic) channel closed in energy is of the form

$$
V=V_{23}+2 V_{21} g_{1}^{0}\left(z-\varepsilon_{1}\right) V_{12}
$$


The system of equations (8) describes the two-channel process of scattering of particle 1 on pair $(2,3)$ vie one-channel interaction of particle 2 (or 3 ) with a pair of particles 1 and 3 (or 2). For this purpose, using system (8), we express $K^{e l}$ in terms of the Green function of the closed (inelastic) channel $g_{v}(x)=\left(x-h_{2}^{0}-V\right)^{-1}$ :

$$
K^{e l}=2 V_{12} g_{v}\left(z-\varepsilon_{2}\right) V_{21},
$$

whose spectrum determines specific features of the elastic channel. Resonances in elastic scattering correspond to points of the spectrum $E_{t}$ of the Hamiltonian $h_{v}=$ $h_{2}^{0}+V$ when $E_{t}>\varepsilon_{1}-\varepsilon_{2}$. Though this statement is obvious, we present the scheme of construction of the S-matrix for resonances because of their peculiarities in the system under consideration. To this end, we consider the case when the energy $z$ is close to $\varepsilon_{2}+E_{t}$ and separate the singular part

$$
g_{v}\left(z-\varepsilon_{2}\right)=\frac{\left|\Psi_{t}><\Psi_{t}\right|}{\omega}+g_{R}
$$

from the Green function $g_{v}$, where $\omega=z-\varepsilon_{2}-E_{t}, \Psi_{t}$ is the wave function corresponding to the eigenvalue $E_{t}$, and $g_{R}$ is a regular part of the Green function usually neglected. Then, using expression (10) and connection of the physical amplitude with $K^{e l}$ (6), we derive the $S$-matrix $\left(S=1+2 i k_{0} f^{e l}\left(k_{0}, k_{0}\right)\right)$ in the form

$$
S=\frac{1-i B}{1+i B} \frac{\omega+\frac{1}{2} \frac{\Gamma B}{1+B^{2}}-i \frac{1}{2} \frac{\Gamma}{1+B^{2}}}{\omega+\frac{1}{2} \frac{\Gamma B}{1+B^{2}}+i \frac{1}{2} \frac{\Gamma}{1+B^{2}}}
$$

where the width

$$
\Gamma=2 \frac{k_{0} m_{1,23}}{\pi}\left|<k_{0}\right| V_{12}\left|\Psi_{t}>\right|^{2}
$$

generated by the singular part of the Green function is changed by the regular part: $B=\frac{k_{0} m_{1,23}}{\pi}<k_{0}\left|V_{12} g_{R} V_{21}\right| k_{0}>$. The shift of a resonance from $E_{t}$ is also determined by that part. It is just the quantity $B$ that determines the background scattering far off the resonance when $S=\frac{1-i B}{1+i B}$. Introducing the background scattering phase $\delta_{f}=-\arctan (\mathrm{B})$, we can rewrite the $S$-matrix in the form

$$
S=\exp \left(2 i \delta_{f}\right) \frac{\omega-i \frac{\Gamma}{4}-\frac{\Gamma}{4}\left(\sin \left(2 \delta_{f}\right)+i \cos \left(2 \delta_{f}\right)\right)}{\omega+i \frac{\Gamma}{4}-\frac{\Gamma}{4}\left(\sin \left(2 \delta_{f}\right)-i \cos \left(2 \delta_{f}\right)\right)} .
$$

It is to be noted that expression (12) has been derived without approximations and is a convenient representation of the $S$-matrix. However, the Breit-Wigner parametrization, separation of the resonance energy and width, imposes a certain constraint on widths. They should be rather small so that an isolated resonance could be considered. The true width of the resonance $\tilde{\Gamma}$ is determined not only by $\Gamma$, but also by the phase of background scattering: $\tilde{\Gamma}=\Gamma \cos ^{2}\left(\delta_{f}\right)$. Just for this reason the expression for $\Gamma$ contains plane waves rather than the wave functions of the state scattering in a certain background potential. Below, we consider an expression for $\Gamma$ that is always larger than or equal to $\tilde{\Gamma}$. Other specific properties of the $S$-matrix represented by (12) are rather obvious and their discussion goes beyond the scope of this paper. 


\section{Wave function of the closed channel}

We are interested in a series of resonances corresponding to the Efimov effect, i.e. when conditions (2) hold valid and the spectrum of the Hamiltonian $h_{v}$ condenses around zero. In this case the potential $V$ gets much simplified, and for studying underthreshold resonances it is sufficient to consider the $S$-part of the effective potential at low energies, or more precisely, at momenta $k \gg \sqrt{\left(-2 m_{2,13} z\right)}$, where solutions to the Schrödinger equations slightly depend on energy. Under this auxiliary condition $(\beta \rightarrow \infty, z \rightarrow 0)$ the terms of the effective potential (9), the "exchange" potential $V_{e x} \equiv V_{23}$ and two "triangular" (with internal integration) potentials $V_{t r} \equiv V_{21} g_{1}^{0}\left(z-\varepsilon_{1}\right) V_{21}$, can be written in the form:

$$
\begin{gathered}
V_{e x}^{0}\left(k, k^{\prime}\right)=-\frac{\pi}{2 \sqrt{k k^{\prime}} \lambda_{1}} \frac{1}{\sqrt{m_{3,12} m_{12}}} \ln \left(\frac{k^{2}+k^{\prime 2}+2 \lambda_{1} k k^{\prime}}{k^{2}+k^{\prime 2}-2 \lambda_{1} k k^{\prime}}\right), \\
V_{t r}^{0}\left(k, k^{\prime}\right)=-\frac{1}{\sqrt{k k^{\prime}}\left(2 \lambda_{1}\right)^{2}} \frac{m_{12}}{m_{23}^{2}} \text { v.p. } \int_{0}^{\infty} \frac{d t}{t-a} L(t, k) L\left(t, k^{\prime}\right) ; \\
L(t, k)=\ln \left(\frac{\gamma t^{2}+k^{2}+2 \lambda_{2} k t}{\gamma t^{2}+k^{2}-2 \lambda_{2} k t}\right), a=\kappa_{1} \sqrt{\frac{m_{1,23}}{m_{32}}}, \lambda_{i}=\sqrt{\frac{m_{i j} m_{i k}}{m_{i}^{2}}}, \gamma=\sqrt{\frac{m_{23}}{m_{12}}} .
\end{gathered}
$$

The upper index 0 means that the effective interaction is considered at $z=0$. The exchange potential $V_{e x}^{0}$ describes the scattering of particle 2 (or 3 ) on a bound pair of particles 1 and 3 (or 2) at the zeroth binding energy in the pair and is a "classical" potential generating Efimov states. Solutions in the field of that potential investigated for a three-boson system [7], coincide with solutions in the field of a local potential of the type $-\left(\mu^{2}+0.25\right) / 2 m_{2,13} \rho^{2}$ ( $\rho$ is the Jacobi coordinate of relative motion of a particle and a pair) with the coupling constant $\mu$ obeying a transcendental equation. Below, we derive an equation of that sort for the total interaction potential. Note that the potential $V_{e x}^{0}$ behaves like $1 / k$ and admits a solution in the form $k^{s}$. The potential $V_{t r}^{0}$ is more complicated in form and admits solutions like that only in the region of momenta where the quantity $a$ in the denominator of the integrand of expression (14) can be neglected. To verify this, we look for solutions to the Schrödinger equation with the zeroth energy in the form $\Psi_{t}=k^{i \mu-5 / 2}$. To this end, we define the quantity $\Pi$ :

$$
\Pi(k)=-\frac{2 m_{2,13}}{k^{i \mu-1 / 2}} \int V^{0}(k, t) t^{i \mu-5 / 2} \frac{d^{3} t}{(2 \pi)^{3}},
$$

and write it with an index corresponding to the potential. This quantity represents the ratio of contributions of the potential and kinetic energies to the Schrödinger equation. Then, the Schrödinger equation is written in the form: $\Pi_{e x}+2 \Pi_{t r}=1$. The contribution of the exchange potential is expressed via the integral

$$
\Pi_{e x}=\frac{1+\zeta_{1}^{2}}{\zeta_{1}} I_{1}, \quad I_{1}=\frac{1}{2 \pi} \int_{0}^{\infty} x^{i \mu-1} \ln \left(\frac{1+2 \lambda_{1} x+x^{2}}{1-2 \lambda_{1} x+x^{2}}\right) d x
$$


$\left(\zeta_{i}=\sqrt{\frac{m_{j} m_{k}}{m_{i} M}}, M\right.$ is the total mass) that exists at $-1<\operatorname{Im} \mu<1$ and is taken over residues upon integrating by parts:

$$
I_{1}=\frac{\operatorname{sh}\left(\mu \arctan \zeta_{1}\right)}{\mu \operatorname{ch}\left(\frac{\pi}{2} \mu\right)}
$$

Introducing the function $\Phi$ in the form

$$
\Phi_{i}(\mu)=\frac{1+\zeta_{i}^{2}}{\zeta_{i}} \frac{\operatorname{sh}\left(\mu \arctan \zeta_{i}\right)}{\mu \operatorname{ch}\left(\frac{\pi}{2} \mu\right)}
$$

we obtain

$$
\Pi_{e x}=\Phi_{1}(\mu) .
$$

The function $\Pi_{t r}(k)$ is, upon simple transformations, expressed in terms of the function $\Phi$ and a new function $\tilde{\Phi}$ :

$$
\begin{gathered}
\Pi_{t r}(k)=\Phi_{2}(\mu) \tilde{\Phi} \\
\tilde{\Phi}=\frac{1+\zeta_{2}^{2}}{2 \pi \zeta_{2}} \text { v.p. } \int_{0}^{\infty} x^{i \mu-1} \frac{k x}{k x-a \gamma} \ln \left(\frac{1+2 \lambda_{2} x+x^{2}}{1-2 \lambda_{2} x+x^{2}}\right) d x .
\end{gathered}
$$

This integral is easily transformed into contour integrals along two logarithmic cuts and integration contours can always be chosen so that either $|x| \geq 1$ or $|x| \leq 1$. This allows decomposition of the denominator of the integrand in a series either at large $(k>a \gamma)$ or at small momenta $(k<a \gamma)$. In the first case, we obtain the leading term $\tilde{\Phi}=\Phi_{2}$; whereas in the second, $\tilde{\Phi} \propto k / \kappa_{1}$. Therefore, we can write the transcendental equations for $\mu$ in two asymptotic regions. Considering that $\Pi_{e x}+2 \Pi_{t r}=1$, we get

$$
\begin{gathered}
\Phi_{1}(\mu)=1, \quad k \ll \sqrt{\frac{m_{1,23}}{m_{12}}} \kappa_{1} ; \\
\Phi_{1}(\mu)+2 \Phi_{2}^{2}(\mu)=1, \quad k \gg \sqrt{\frac{m_{1,23}}{m_{12}}} \kappa_{1} .
\end{gathered}
$$

Owing to the function $\Phi$ being even in $\mu$, the wave function $\Psi_{t}$ is a linear combination of $k^{ \pm i \mu-5 / 2}$, thus coinciding with the Fourier transform of the wave function in the field of the local potential

$$
V_{e f}(\rho)=-\frac{\mu^{2}+0.25}{2 m_{2,13} \rho^{2}}
$$

when $|V| \gg|z|$, with different coupling constants at small and large $\rho$. Solutions in the field of $1 / \rho^{2}$-type potential are well known. Specifically, the energy levels obey the relation $E_{n-1} / E_{n}=\eta$, where the quantity $\eta$ depends merely on $\mu$ :

$$
\eta=\exp (2 \pi / \mu)
$$

and they either go to minus infinity (fall onto the center indicated by Thomas [9], or are condensed to zero from below (the Efimov effect [4]). In our case, deep levels correspond to small distances and are determined by the value of $\mu_{2}$ that obeys the 
equation (16). Shallow levels with $z>\varepsilon_{1}$ in the scattering channel produce resonances and are determined by the value of $\mu_{1}$ from equation (15).

A simple analysis of eqs.(15) and (16) shows that $\mu_{2}$ exponentially tends to $\mu_{1}$ when $m_{1} / m_{2} \rightarrow 0$, and the quantity $\mu_{1}$ in this limit is described by the expansion:

$$
\mu_{a s}=c \zeta_{1}+\frac{c}{(c+1) \zeta_{1}}+O\left(\frac{1}{\zeta_{1}^{3}}\right)
$$

$\left(\zeta_{1}=\sqrt{m_{2} m_{3} / m_{1} M} \gg 1\right)$, coinciding with the limit found earlier [1]. The constant $c=0.5671 \ldots$ obeys the equation $c=\exp (-c)$. We do not present detailed computations and cite the dependences of quantities $\mu$ on the ratio of masses of a light and a heavy particle in Table 1. Due to the rapid convergence of $\mu_{2}$ to $\mu_{1}$, the second term in eq. (16) gets negligible (solutions differ in the sixth digit at $m_{2} / m_{1}=100$ ), and thus, in the limit $m_{1} / m_{2} \ll 1$ the second term in the potential $V$ can be neglected. To demonstrate the smallness, we present the ratio of potential energies $\Pi_{e x}\left(\mu_{1}\right)$ and $\Pi_{t r}\left(\mu_{1}\right)$.

Table 1: The dependences of $\mu_{1}, \mu_{2}, \mu_{a s}$ on masses (for explanation, see the text)

\begin{tabular}{|c|c|c|c|c|c|c|}
\hline$m_{2} / m_{1}$ & 10 & 20 & 30 & 50 & 70 & 100 \\
\hline$\mu_{1}$ & 1.379051 & 1.893909 & 2.284713 & 2.906777 & 3.415826 & 4.061110 \\
$\mu_{2}$ & 1.468174 & 1.919444 & 2.293865 & 2.908434 & 3.416219 & 4.061172 \\
$\mu_{a s}$ & 1.430016 & 1.907906 & 2.289978 & 2.908096 & 3.416437 & 4.061489 \\
$\Pi_{t r} / \Pi_{e x}$ & $1.1210^{-1}$ & $2.2010^{-2}$ & $6.4110^{-3}$ & $9.0110^{-4}$ & $1.8110^{-4}$ & $2.3810^{-5}$ \\
\hline
\end{tabular}

Thus, the wave function $\Psi_{t}$ for a very light particle 1 in a large region $(k \gg$ $\left.\sqrt{\left(-2 m_{2,13} z\right)}\right)$ of momenta is a linear combination of the functions $k^{ \pm i \mu-5 / 2}$ with the quantity $\mu=\mu_{1}$. This momentum asymptotics is sufficient for computing the matrix elements in the width $\Gamma$ given by (11) since a dominant contribution to these integrals comes from the region of momenta $\sim \kappa_{1}$. The difficulty in normalizing the wave function can be overcome in a simple manner. Our wave function describes the motion in the effective potential (17). Solutions in the field of that potential are well known: $\Psi_{t}(\rho) \sim$ $K_{i \mu} / \sqrt{\rho}$, they are expressed in terms of modified Bessel functions. The function $\Psi_{t}$ can be normalized, its Fourier transform and coefficients of the leading asymptotic terms $k^{ \pm i \mu-5 / 2}$ can be obtained. This scheme is somewhat cumbersome but produces quite a reasonable result that for a small mass of particle 1 coincides with the exact result. The latter (at zeroth two-body forces) can be obtained more easily: the solution to the integral equation with potential $V_{e x}$ can be derived in the whole region of momenta, without setting $z=0$, by the Mellin transformation, like it has been carried out in refs. [10, 11] in studying the properties of the Skornyakov-Ter-Martirosyan equation [12]. In ref. [11], an approximate wave function has been found for a system of three bosons with zeroth two-body forces which at zeroth binding energies in subsystems becomes exact. The Lippmann-Schwinger equation with potential $V_{e x}$ throughout the whole momentum region for the function $\psi=k\left(k^{2}-2 m_{2,13} z\right)^{3 / 4} \Psi_{t}$, upon making $t=k / \kappa_{t}\left(\kappa_{t}=\sqrt{-2 m_{2,13} z}\right)$ to be dimensionless, differs from the Skornyakov-TerMartirosyan for three bosons by factor 2 and different values of the mass constants $\lambda_{1}$ 
and $\alpha=\sqrt{m_{13} / m_{2,13}}$ :

$$
\psi(t)=\frac{1}{2 \pi \lambda_{1} \alpha} \int_{0}^{\infty} d t^{\prime} \ln \left(\frac{t^{2}+t^{\prime 2}+2 \lambda_{1} t t^{\prime}+\alpha^{2}}{t^{2}+t^{\prime 2}-2 \lambda_{1} t t^{\prime}+\alpha^{2}}\right) \frac{1}{\sqrt{t^{\prime 2}+1}} \psi\left(t^{\prime}\right) .
$$

Therefore, equation (20) has also an analytic equation that can be derived by the Mellin transformation. Here we present the final result:

$$
\psi(t)=A \sin \left(\mu \ln \left(t+\sqrt{1+t^{2}}\right)\right),
$$

that can be verified by a simple substitution of $\psi$ into eq. (20). The constant $\mu$ again obeys equation (15), and the constant $A$ is defined by normalization. The integral equation (20) is solvable at all energies, however, the spectrum can be fixed by imposing constraints on the coefficients of $\sin (\mu \ln k)$ and $\cos (\mu \ln k)$ at large $k$ [10, 11]:

$$
\sin \left(\mu \ln \kappa_{t}\right)=b \cos \left(\mu \ln \kappa_{t}\right) .
$$

An arbitrary constant $b$ determines the wave number $\kappa_{t}^{(n)}$ for a level with number $n$ :

$$
\kappa_{t}^{(n)}=\exp (\pi n / \mu+\arctan (\mathrm{b}) / \mu)
$$

The spectrum thus found satisfies equation (18) and demonstrates the effect of fall on the center at large positive $n$, noted in ref. [11], and the Efimov effect (logarithmic condensation of levels around $z=0$ ) at large negative $n$, later discovered by a different method 《4.

Normalizing the wave function $\Psi_{t}$ over the whole momentum space, we arrive at the final result for the wave function of the closed channel:

$$
\Psi_{t}(k)=\frac{2 \pi}{\sqrt{\kappa_{t}^{3}(1-\pi \mu / \operatorname{sh}(\pi \mu))}} \frac{1}{t\left(1+t^{2}\right)^{3 / 4}} \sin \left(\mu \ln \left(t+\sqrt{1+t^{2}}\right)\right) .
$$

\section{Widths of underthreshold resonances}

As mentioned above, the width of a resonance $\tilde{\Gamma}$ depends on the phase of background scattering and on $\Gamma$ (11) that is defined via matrix elements of the potential $V_{12}$ that connects the open and closed channels of elastic scattering. The background phase depends on details of short-range two-body forces and cannot be considered (has no limit) at the zeroth ranges of the forces, whereas the quantity $\Gamma$, being an upper limit of the resonance width, can in this limit $(\beta \rightarrow \infty)$ be calculated. In view of the equality $E_{k}+\varepsilon_{1}=E_{r}$ for low $\left|E_{r}\right|$ (as compared to $\left|\varepsilon_{1}\right|$ ) we can set $k_{0} \rightarrow \kappa_{1} \sqrt{m_{1,23} / m_{23}}$. Owing to a simple relation $E_{r}=E_{t}+\Gamma \sin \left(2 \delta_{f}\right) / 4$ (see (12)) and small widths $\Gamma$, in the expressions given below we put $E_{r}=E_{t}$. Then considering the definition of $V_{12}$ and the width given by (11), trivial computations result in the expression

$$
\frac{\Gamma}{\left|E_{t}\right|}=32 \pi\left(\frac{m_{2,13}}{m_{23}}\right)^{2}|J|^{2}
$$


where $J$ is given by the integral over the whole momentum space:

$$
J=\frac{\kappa_{1}}{\kappa_{t}} \int \frac{d^{3} k}{(2 \pi)^{3}} \frac{\left(k^{2}+\kappa_{t}^{2}\right)^{1 / 4}}{\kappa_{1}^{2}+k^{2}-\mathbf{k}_{\mathbf{0}} \mathbf{k}+\frac{1}{4} k_{0}^{2}} \Psi_{t}(k) .
$$

By using the wave function of the closed channel (21), we can compute this integral analytically. Since this procedure is quite complicated, we here only outline it. After a trivial angular integration and making $t=k / \kappa_{t}$ dimensionless, we should make the change of variables $t=\left(x^{2}-1\right) /(2 x)$ that allows us to get rid of radicals and transforms the integration range from $(0, \infty)$ to $(1, \infty)$. The integrand is invariant under the transformation $x \rightarrow 1 / x$, which permits a back change to the interval $(0, \infty)$. Upon integration by parts we get rid of the logarithmic function, and the integral $I$

$$
I=\int_{0}^{\infty} x^{i \mu} \frac{Q_{1}(x)}{Q_{2}(x)} d x
$$

where $Q_{1}$ and $Q_{2}$ are polynomials providing convergence at zero and infinity, is computed in a standard way, the integral over the upper edge of the power cut is expressed via a contour integral along the cut. The latter can be closed at infinity and expressed in terms of residues at zeros of the polynomial $Q_{2}$.

Upon that integration, we obtain the following expression for $J$ :

$$
J=\frac{1}{2 \sqrt{1-\pi \mu / \operatorname{sh}(\pi \mu)}} \frac{\operatorname{sh}\left(\mu \arctan \left(\zeta_{2}\right)\right)}{\mu \zeta_{2}} \frac{\sin \left(\mu \ln \left(2 \sqrt{\varepsilon_{1} / E_{t}}\right)\right)}{\operatorname{ch}(\pi \mu / 2)} .
$$

Note remarkable properties of expressions (22) and (23). First, the relative width $\Gamma /\left|E_{t}\right|$ does not depend on the number of a resonance as positions of resonances are connected by expression (18), as a result of which for resonances with numbers $n_{i}$ and $n_{i+k}$ the quantity $J$ differs by a factor $(-1)^{k}$. Therefore, on the logarithmic scale, not only the positions of resonances but also their widths are equidistant. Second, the relative width of resonances exponentially decreases with the mass of particle 1 . To verify this, we write a limiting expression $\left(m_{1} / m_{2} \ll 1\right)$ for the relative width. Considering (19) and that

$$
\left(\frac{\operatorname{sh}\left(\mu \arctan \left(\zeta_{2}\right)\right)}{\mu \zeta_{2}}\right)^{2} \rightarrow 1.024 \ldots
$$

that limit can be represented in the form

$$
\frac{\Gamma}{\left|E_{t}\right|} \simeq 32 \pi 1.024 \exp \left(-\pi c \sqrt{\frac{m_{2}}{2 m_{1}}}\right) \sin ^{2}\left(\frac{\mu}{2}\left(\ln 4 \frac{\varepsilon_{1}}{E_{t}}\right)\right) .
$$

As the observed width $\tilde{\Gamma}$ is always smaller than $\Gamma$, we can make an upper limit on the width of a threshold resonance

$$
\tilde{\Gamma}<103.0\left|E_{r}\right| \exp \left(-1.260 \sqrt{\frac{m_{2}}{m_{1}}}\right)
$$


where we go back to the notation $E_{r}$ since the difference between $E_{t}$ and $E_{r}$ is exponentially small. With expression (25), we can estimate lifetimes of strongly excited negative ions of two-atomic molecules. Already for the proton-electron mass ratio and certainly overestimated scale of the binding energy of subsystem $\left(\left|E_{r}\right|\right)$ in $1 \mathrm{eV}$, the lifetime of a resonance $(1 / \tilde{\Gamma})$ is larger than $10^{6} c$ and exceeds all possible relaxation times in gases. More interesting are systems of a low energy of affinity of an electron and an atom. As mentioned in the Introduction, these systems can be negative ions of molecules of alkali metals. Inserting the mass ratio for lithium into expression (25) we obtain the lifetime $10^{40} c$ much greater than that of the Universe.

\section{Numerical solutions}

To demonstrate the validity of analytic computations, we numerically solve the system of Faddeev integral equations ([) describing the scattering of particle 1 on a pair of identical particles (2 and 3). Computations were performed by the scheme described earlier [5] in which scattering can be considered as close as possible to the reaction threshold. Our calculations do not require such maximal proximity since the coupling

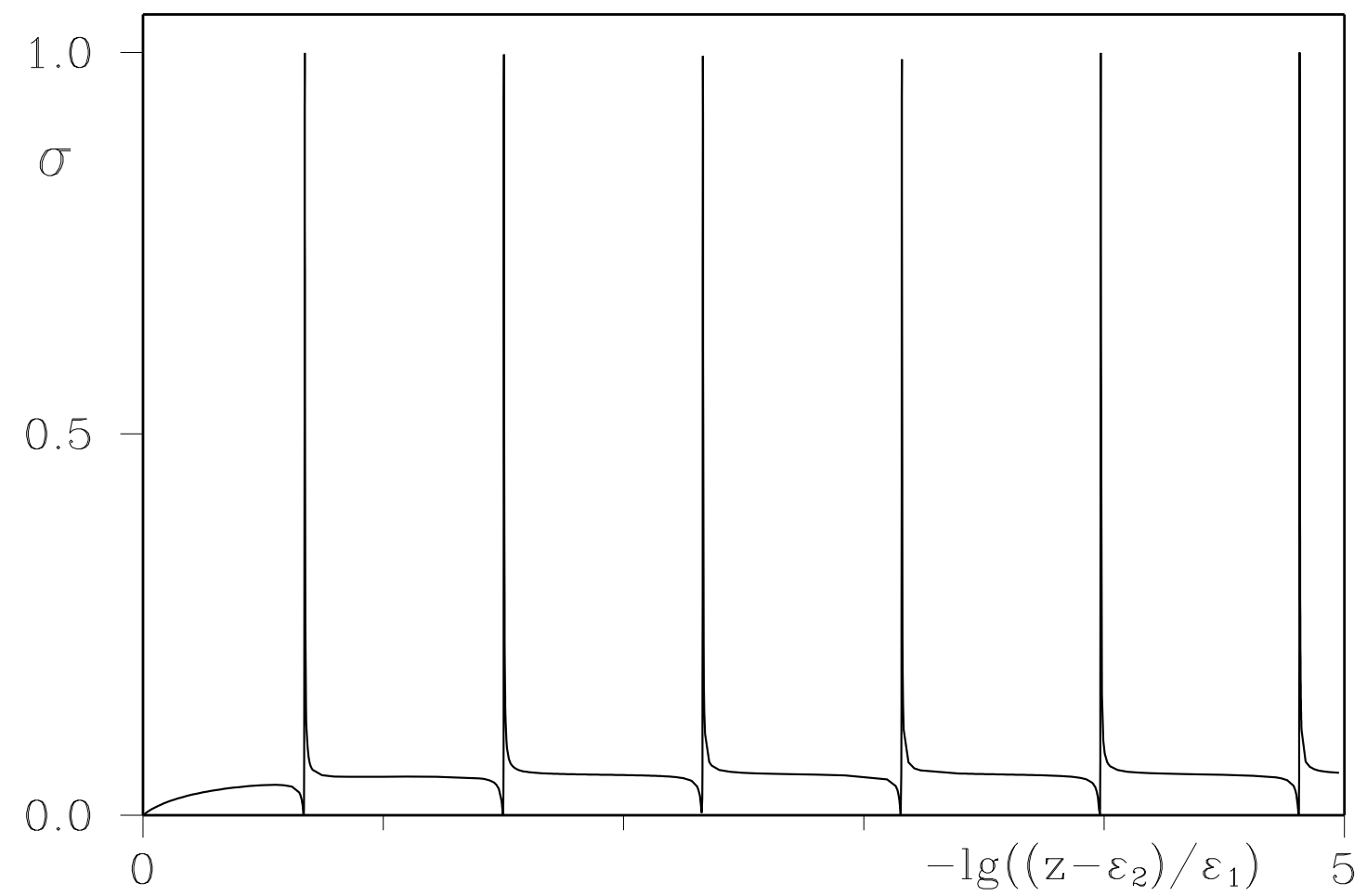

Figure 1: Scattering cross sections for $m_{2} / m_{1}=65$. Explanations are given in the text

$\mu$ is large and we restrict our consideration to the region from zero kinetic energy $\left(z=\varepsilon_{1}\right)$ up to an energy being remote from the rearrangement threshold by $10^{-8} \mathrm{MeV}$ 
$\left(z-\varepsilon_{2}=-10^{-8} \mathrm{MeV}\right)$. The rearrangement threshold differs from the three-particle one by $10^{-15} \mathrm{MeV}\left(\varepsilon_{2}=-10^{-15} \mathrm{MeV}\right)$, and the binding energy in pair $(2,3) \varepsilon_{1}$ equals $-10^{-3}$ $\mathrm{MeV}$. The masses of particles 2 and 3 are equal to the nucleon mass. The constant $\beta$ that determines the interaction range is taken the same for all two-body potentials and equals $0.72 \mathrm{fm}^{-1}$. Measurement units for the energy $(\mathrm{MeV})$ and distances (fm) are taken in accord with the Yamaguchi potential with parameters of the nuclear problem (masses and interaction ranges) and characteristic potential energy in several ten $\mathrm{MeV}$. So, numerical calculations were based on small but finite interaction ranges of two-body forces and binding energies in pairs. As the problem is completely specified by the ratio of dimensional quantities, the results of calculations are also valid for atomic units. For instance, conditions (2) for pairs

$$
\kappa_{1} / \beta_{1} \simeq 6.7 \cdot 10^{-3} ; \quad \kappa_{2} / \beta_{2}=10^{-6} \sqrt{m_{1} / m_{2}} \kappa_{1} / \beta_{1},
$$

on the atomic scale of interaction ranges of two-body forces in $1 \AA\left(r_{0} \simeq 3 / \beta\right)$ correspond to the length of atom-atomic scattering $\left(1 / \kappa_{1}\right)$ in about $50 \AA$.

In Fig.1, we plot the scattering cross sections normalized to the unitary limit in Swave, $\sigma_{u n}=4 \pi / k_{0}^{2}$, for the mass ratio $m_{2} / m_{1}=65$. Since resonances condense to $\mathrm{z}=0$, the energy is plotted on the logarithmic scale. From Fig. 1 one can clearly observe the equidistance of resonance positions. Unfortunately, even the maximum width in this mass region of particle 1 (see Fig.2) remains very small and resonances are practically straight lines.

In Table 2 we report positions of the first six resonances, the ratio of the energy of a preceding resonance to that with a given number, i.e., the quantity $\eta_{\text {calc }}$, and relative widths of resonances. For comparison, we present the values of $\mu_{1}$ and $\mu_{2}$, solutions to equations (15) and (16), respectively: $\mu_{1}=3.29589 \ldots, \mu_{2}=3.29654 \ldots$. They correspond to the values of $\eta$ given by (18)): $\eta\left(\mu_{1}\right)=6.72823 \ldots$ and $\eta\left(\mu_{2}\right)=6.72608 \ldots$. From the Table it is seen tat the energy ratio of the second to the third resonance

Table 2: Parameters of the first six resonances at $m_{2} / m_{1}=65$. Explanations are given in the text

\begin{tabular}{|c|c|c|c|}
\hline$N$ & $E_{r} / \varepsilon_{1}$ & $\eta_{\text {calc }}$ & $\Gamma /\left|E_{r}\right|$ \\
\hline 1 & $2.1236 \quad 10^{-1}$ & & $4.41310^{-3}$ \\
2 & $3.148010^{-2}$ & 6.746 & $4.246 \quad 10^{-3}$ \\
3 & $4.6787 \quad 10^{-3}$ & 6.728 & $4.208 \quad 10^{-3}$ \\
4 & $6.954010^{-4}$ & 6.728 & $4.202 \quad 10^{-3}$ \\
5 & $1.0338 \quad 10^{-4}$ & 6.727 & $4.201 \quad 10^{-3}$ \\
6 & $1.536910^{-5}$ & 6.726 & $4.200 \quad 10^{-3}$ \\
\hline
\end{tabular}

is in the region of analytic values of $\eta$ up the fourth decimal point. Note that the first resonance is quite far from the threshold. The last column demonstrates the relative widths to be independent of the number of a resonance. Starting from the 
third resonance, changes occur in the fourth decimal place. So, Figure 1 and Table 2 confirm the logarithmic equidistance of the positions of resonances and their widths that manifests itself in the constant character of relative widths of resonances.

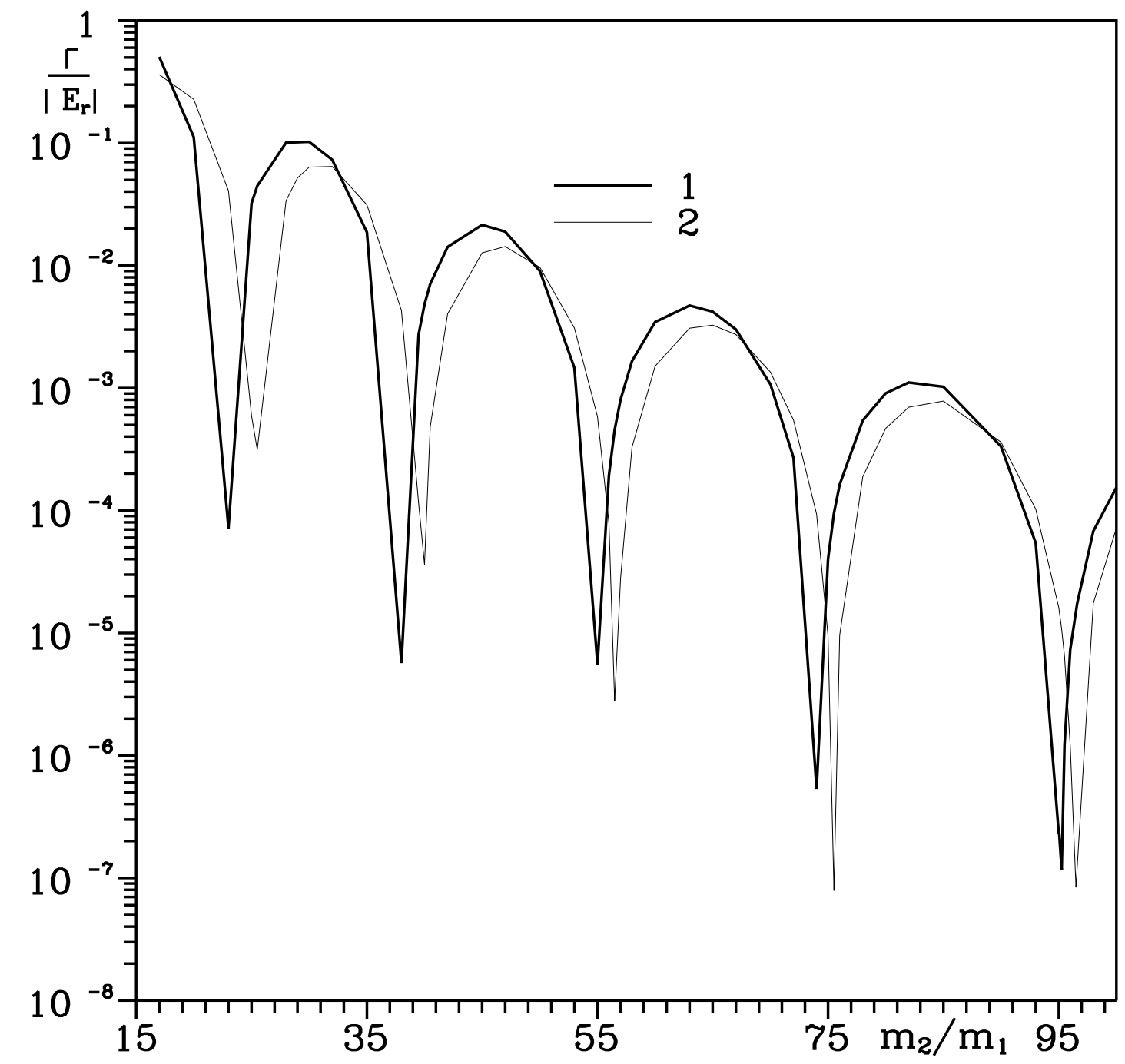

Figure 2: Relative widths of resonances. 1 is a numerical solution of the Faddeev equations; 2, the analytic dependence. Explanations are given in the text.

To demonstrate the validity of expressions (22) and (23) in describing the analytic connection of widths of resonances and their positions at small ratios of masses $m_{1} / m_{2}$, in Fig. 2 we draw the relative widths obtained from numerical solutions to integral equations (a solid curve) and from expression (22) by substituting numerically found values of resonance energies into it (a thin curve). Analytic dependence of the resonance widths on masses follows the curve though it has a small phase shift. Note some peculiarities of the dependence of widths on the mass of a light particle 1. First, the width of a resonance is not a monotone function of its mass. Second, the maximum 
of the widths of resonances exponentially decrease with the mass of particle 1 in accordance with the estimation (24). In particular, for the range of mass changes $\left(m_{2} / m_{1}\right)$, from 15 to 100, shown in the Figure, the widths of resonances fall by three decimal orders. A strong nonmonotone character of relative allows us to see the difference of analytic estimations from the numerical results. If we estimate on the basis of sharp local minima, then the observed phase shift for the minimum amount to 2 mass units and monotonically decreases to 1.25 units in the region of the last minimum in the Figure $\left(m_{2} / m_{1} \sim 95\right)$. Thus, the difference between analytic and numerical estimates diminishes with mass $m_{1}$.

\section{Conclusion}

So, we have estimated lifetimes of the Efimov states of negative ions on the basis of the scattering problem of a light particle (an electron) on a bound pair of two heavy particles (molecules) under condition (2). This three-body problem allows one to derive analytic expressions for widths and positions of resonances and at the same time to verify them by a direct numerical solution of the Faddeev equations. For the first time, we have computed Efimov resonances under the threshold of rearrangement of a three-particle system.

Our consideration leads us to conclusion that lifetimes of a new class of molecular states discussed in refs. [1, 2, 3] testify in favour of them being bound states for all types of physical processes. The work was performed within in project ISTC K-40.

\section{References}

[1] F.M.Pen'kov, JETP 79, 568 (1994).

[2] F.M.Pen'kov, Zh. Eksp. Teor. Fiz. 109, 721 (1996).

[3] F.M.Pen'kov, JETP 84, 678 (1997).

[4] V.Efimov, Sov. J. Nuclear Phys. 12, 589 (1971).

[5] F.M.Pen'kov, N.Zh.Takibayev, Yad. Fiz. 57, 1300 (1994).

[6] L.D.Faddeev, S.P.Merkuriev, Quantum Scattering Theory for Several Particle Systems, (Kluwer, Dordrecht, 1993).

[7] N.Zh.Takibayev, F.M.Pen'kov, Sov. J. Nucl. Phys. 50, 234 (1989).

[8] V.B.Belyaev, Lectures on theory for few-body systems (in Russian), (Energoatomizdat, Moscow (1986), p.37).

[9] L.H.Thomas, Phys.Rev. 47, 903 (1935).

[10] G.S.Danilov, Zh. Eksp. Teor. Fiz. 40, 498 (1961). 
[11] R.A.Minlos, L.D.Faddeev, Zh. Eksp. Teor. Fiz. 41, 1850 (1961).

[12] G.V.Skornyakov, K.A.Ter-Martirosyan, Zh. Eksp. Teor. Fiz. 31, 775 (1956). 